\section{New strategies for CNS regeneration}

\section{By Tim Fulmer, Senior Writer}

Two separate teams of company and university researchers have identified multiple neuronal proteins that could be targeted alone or in combination to promote axon growth and treat spinal cord injury and other forms of CNS trauma. ${ }^{1,2}$ The findings could overcome a fundamental obstacle facing efforts to improve neuronal function after injury-the inability of the adult CNS to regenerate.

In one of the two Science papers, researchers at Genentech Inc. and Stanford University reported finding a potential master sensor of extracellular growth inhibition signals that could be targeted to help injured neurons regenerate in adults. The California group focused on blocking interactions between neurons and extracellular myelin-derived growth inhibitory factors (see Figure 1, “Overcoming growth inhibition”).

In contrast, Harvard Medical School scientists focused on activating signaling pathways within neurons to promote axon growth. They identified phosphatase and tensin homolog (PTEN; MMAC1; TEP1) as an intracellular target that could achieve the same result (see Figure 1, “Overcoming growth inhibition").

Myelin and myelin-derived proteins are well-established inhibitors of axon growth in the adult CNS. One of the best studied of these myelin-derived inhibitory factors is reticulon 4 (RTN4; NOGO-A), which binds the reticulon 4 receptor (RTN4R; NgR1; NOGOR) on the surface of neurons and triggers downstream signaling pathways that inhibit nerve fiber growth.

Indeed, the importance of NOGO-A in inhibiting axonal growth has led Novartis AG to start a Phase I trial of an anti-NOGO-A mAb to treat spinal cord injury (SCI).

\section{Funneling regeneration}

The Genentech and Stanford team have found a new player-leukocyte immunoglobulin-like receptor subfamily B member 2 (LILRB2). It binds myelin-derived growth inhibitory factors and interacts with the NOGO-A pathway to inhibit axon growth.

In a cell culture binding assay, LILRB2 and its murine equivalent, paired immunoglobulin-like receptor B (PirB), bound multiple axonal growth inhibitors, including NOGO-A, myelin-associated glycoprotein (MAG) and oligodendrocyte myelin glycoprotein (OMG).

In cultured mouse cerebellar granule neurons grown in the presence of Nogo-A or myelin, a soluble PirB decoy receptor or an anti-PirB antibody significantly increased neurite outgrowth length compared with that seen in untreated control cells $(p<0.01)$.
PirB inhibition in NgR1-deficient neurons led to additional improvements in neurite outgrowth, suggesting that PirB and NgR1 together help mediate myelin-associated growth inhibition.

The authors concluded that blocking LILRB2 in humans "either with antibodies or by other means, provides an important target for therapeutic interventions to stimulate axon regeneration."

"Clearly the key next step is to study the PirB pathway in the adult CNS of preclinical injury models. This should provide insight into the types of axon growth and sprouting that are stimulated by PirB blockade and the distances over which that growth occurs," said Stephen Strittmatter, professor of neurology at Yale University School of Medicine.

Strittmatter and colleagues have a soluble NgR1 decoy receptor that has improved recovery in two different rodent models of SCI and a rodent model of stroke. ${ }^{3-5}$

The Genentech group now plans to look at the effects of PirB knockout and pharmacological inhibition in rodent models of SCI, said Marc Tessier-Lavigne, corresponding author on the paper and EVP of research drug discovery at Genentech.

"We anticipate that targeting a receptor like PirB could have therapeutic advantages over targeting a single ligand like NOGO-A because PirB binds multiple inhibitory ligands in addition to NOGO-A. In a sense, we're blocking the funnel through which all these ligands exert their inhibitory effects rather than antagonizing any single one ligand," added Tessier-Lavigne.

Carla Shatz, a principal investigator on the paper and a professor of neurobiology at Stanford, told SciBX that targeting PirB also could have value in treating cognitive disorders that result from decreased neural plasticity.

In previous work in mice, Shatz and colleagues found that PirB is expressed in subsets of neurons throughout the brain and that adult mice lacking functional PirB have increased neural plasticity in the visual cortex. ${ }^{6}$ "This suggests that PirB normally acts to restrict neural plasticity and that blocking the receptor could help increase the plasticity of some neural circuits and potentially help improve memory loss and learning disorders," she said.

Similarly, Strittmatter and colleagues have found that $\mathrm{NgR} 1$ restricts experience-driven plasticity in the visual cortex. ${ }^{7}$

"These findings strongly suggest that compounds targeting PirB and $\mathrm{NgR} 1$ to enhance neuronal regeneration in spinal cord injury could potentially also be useful for enhancing neural plasticity in cognitive disorders," said Shatz.

"Subsequent studies in PirB knockout mice could help reveal PirB's importance in structural and functional synaptic plasticity of neurons in the adult hippocampus. In particular, the researchers could look at how knocking out PirB affects long-term potentiation, a form of synaptic plasticity that underlies aspects of learning and memory," said Roman Giger, associate professor of neurology at the University of Michigan Medical School.

Giger and colleagues have shown that knocking out NgR1 in mice led to increased activity-dependent synaptic plasticity and better long-term potentiation in the presence of fibroblast growth factor 2 (FGF2). ${ }^{8}$ 
Figure 1. Overcoming growth inhibition.

Two articles in Science illustrate contrasting but potentially complementary strategies to promote axon growth following injury. ${ }^{1,2}$

[a] Genentech Inc. and Stanford

University researchers identified leukocyte immunoglobulin-like receptor subfamily B member 2 (LILRB2), a neuronal surface receptor that mediates the growth inhibitory effects of multiple myelin-associated ligands, including reticulon 4 (RTN4; NOGO-A), myelin-associated glycoprotein (MAG) and oligodendrocyte myelin glycoprotein (OMG).

Previous work by other laboratories has shown a similar role for another neuronal surface receptor, reticulon 4 receptor (RTN4R; NgR1; NOGOR), which acts on the Rho kinase pathway to trigger its growth inhibitory effects. The pathway on which LILBR2 exerts its effects has yet to be elucidated.

[b] In the second paper, researchers MMAC1; TEP1) promoted axon growth in injured retinal ganglion cells.

PTEN is a negative regulator of the prosurvival phosphoinositide 3-kinase (PI3K) and mammalian target of rapamycin (mTOR) pathway. Thus, PTEN inhibitors would be predicted to promote survival and axon growth. Inhibitors of proteins downstream of PTEN, such as tuberous sclerosis 1 (TSC1), could have similar growth-promoting effects. (Phosphatidylinositol-4,5-bisphosphate (PIP ${ }_{2}$ ); phosphatidylinositol-3,4,5-trisphosphate $\left(\mathrm{PIP}_{3}\right)$; protein kinase $\mathrm{B}(\mathrm{Akt})$; ras homolog enriched in brain $(\mathrm{RHEB})$; mammalian target of rapamycin complex 1 (mTORC1).)

\section{Promoting proliferation}

Although neutralization of extracellular growth inhibitors offers one approach to promoting axonal growth, a second and potentially complementary strategy seeks to activate signaling pathways within adult neurons to trigger axonal survival and growth in the wake of injury.

Along these lines, researchers at Harvard Medical School tested whether knocking out genes that prevent cellular overgrowth in adulthood could improve the survival and regenerative capacity of injured adult mouse retinal ganglion cells.

Among the genes deleted in the cells, knocking out PTEN, a tumor suppressor, resulted in the greatest improvements in survival and axonal elongation after crushing the optic nerve. Importantly, the axonal sprouts of PTEN-deficient retinal ganglion cells penetrated into the glial-enriched lesion site, indicating the cells were able to overcome glial-mediated growth inhibition after injury.

PTEN is a negative regulator of the prosurvival phosphoinositide 3kinase (PI3K) and mammalian target of rapamycin (mTOR) pathway. Thus, inhibiting the protein would be expected to increase mTORmediated protein synthesis and cell growth. Indeed, wild-type retinal ganglion cells showed sustained downregulation of mTOR following injury, whereas PTEN-deficient cells maintained mTOR activity at levels similar to those of uninjured wild-type cells.

The authors concluded that their findings "suggest the manipulation of intrinsic growth control pathways as a therapeutic approach to promote axon regeneration after CNS injury."

"Key next steps now include looking at PTEN deletion in rodent models of spinal cord injury, which typically have more inflammation and glial scarring than what occurs following optical nerve crush," said Zhigang He, corresponding author on the paper, research associate at the Children's Hospital Boston and associate professor of neurobiology at Harvard Medical School. "Those studies should also help establish whether PTEN blockade results in functional recovery of neuronal connections beyond improving their capacity to regenerate axons."

He and colleagues are collaborating with Sheng Ding at The Scripps Research Institute to develop small molecule inhibitors of PTEN and its downstream effectors in the PI3K/mTOR pathway, such as tuberous sclerosis 1 (TSC1). 
"The mTOR pathway potentially provides multiple nodal points for targeted intervention to promote regeneration," Ding told SciBX. "Options include inhibiting negative regulators like PTEN and TSC1 or directly activating pathway components like PI3K or mTOR. In any case, the key will be to avoid sustained, long-term activation of this pathway, which would likely be oncogenic given that PTEN is a tumor suppressor."

Ding is associate professor of chemistry and cell biology at Scripps. His lab focuses on using high throughput screens to identify small molecules that stimulate endogenous cells to regenerate and differentiate. ${ }^{9}$

"Activating PTEN in order to downregulate PI3K and thus block cell proliferation has been the strategy in cancer. However, there is mounting evidence that inhibiting PTEN to increase proliferation and generate a prosurvival effect could have therapeutic value in the cell regeneration setting," said Joseph Garlich, CSO of Semafore Pharmaceuticals Inc., a biotech focused on developing small molecule modulators of PTEN and PI3K signaling.

Garlich did say the Harvard researchers need to work out the proper treatment duration and dosing regimen for the PTEN inhibition strategy in animal models. "This is critical to ensuring that a small molecule PTEN inhibitor promotes regeneration in the appropriate cells without also triggering an oncogenic signal," he said.

"Ultimately, the ideal strategy for promoting axon regrowth in the adult CNS could be some form of combination therapy that unites approaches targeting extracellular factors like NOGO-A with approaches targeting intrinsic factors like PTEN," said He.

The PTEN inhibition strategy to promote axonal regeneration is patented and available for licensing through Harvard Medical School.

Fulmer, T. SciBX 1(43); doi:10.1038/scibx.2008.1036

Published online Dec. 4, 2008

REFERENCES

1. Park, K. et al. Science; published online Nov. 7, 2008; doi:10.1126/science.1161566

Contact: Zhigang He, Harvard Medical School, Boston, Mass. e-mail: zhigang.he@childrens.harvard.edu

2. Atwal, J. et al. Science; published online Nov. 7, 2008; doi:10.1126/science.1161151

Contact: Marc Tessier-Lavigne, Genentech Inc., South San Francisco, Calif. e-mail: marct|@gene.com

3. Ji, B. et al. Eur. J. Neurosci. 22, 587-594 (2005)

4. Wang, X. et al. Ann. Neurol. 60, 540-549 (2006)

5. Lee, J. et al. J. Neurosci. 24, 6209-6217 (2004)

6. Syken, J. et al. Science 313, 1795-1800 (2006)

7. McGee, A. et al. Science 309, 2222-2226 (2005)

8. Lee, H. J. Neurosci. 28, 2753-2765 (2008)

9. Xu, Y. \& Ding, S. Nature 453, 338-344 (2008)

COMPANIES AND INSTITUTIONS MENTIONED

Children's Hospital Boston, Boston, Mass. Genentech Inc. (NYSE:DNA), South San Francisco, Calif. Harvard Medical School, Boston, Mass. Novartis AG (NYSE:NVS; SWX:NOVN), Basel, Switzerland The Scripps Research Institute, La Jolla, Calif. Semafore Pharmaceuticals Inc., Indianapolis, Ind. Stanford University, Stanford, Calif.

University of Michigan Medical School, Ann Arbor, Mich. Yale University School of Medicine, New Haven, Conn. 